\title{
In Situ Studies of Molecular Transfer between Microparticles by Second-Harmonic Generation
}

\author{
Elsa C. Y. Yan, Yan Liu, and K. B. Eisenthal* \\ Chemistry Department, Columbia University, New York, New York 10027
}

Received: May 21, 2001

\begin{abstract}
We have demonstrated that second-harmonic generation (SHG) can be used to investigate the in situ transfer of molecules between colloidal microparticles. The transfer kinetics of organic cations, malachite green (MG) initially adsorbed on to spherical polystyrene sulfate (PSS) microparticles, to disk-shaped clay particles in aqueous solution was obtained. The surface population of MG on the PSS particles during the transfer process can be measured in real time. In the present studies, the adsorption free energy and the number of adsorption sites were found from the adsorption isotherm measured by the SHG method (Wang, H.; Yan, E. C. Y.; Liu, Y.; Eisenthal, K. B. J. Phys. Chem. B 1998, 102, 4446). The kinetics of transfer was observed to depend linearly on the particle density of clay. The observed transfer was biexponential with decay times of $17 \pm 1$ $\mathrm{s}$ and $217 \pm 4 \mathrm{~s}$ for the case of a clay particle density of $3 \times 10^{9} / \mathrm{cm}^{3}$. This method was further applied to study the transfer of MG between PSS microparticles. At a PSS density of $4 \times 10^{8} / \mathrm{cm}^{3}$, the decay time was $40 \mathrm{~s}$. Because of the coherent nature of the SHG signal, the transfer kinetics can be obtained in situ by the SHG method. A kinetic model is used to describe the transfer process from which the desorption and adsorption rate constants of MG from and onto PSS particles were obtained.
\end{abstract}

\section{Introduction}

Colloidal microparticles are scientifically as well as technologically important. Molecular transfer between these microscopic particles is important in various physical, chemical, and biological processes. In the present studies, the second-harmonic generation (SHG) method was applied for the first time to study molecular transfer between colloidal microscopic particles suspended in aqueous solutions. The transfers of triphenyl cations, malachite green (MG), Scheme 1, from polymer particles to clay particles and from the polymer particles to polymer particles of the same kind in aqueous solutions were studied by the SHG method. Clay particles have significant environmental importance because of their abundance in nature. The diffusion of environmental pollutants is related to the rate of uptake of molecules from the solution by the clay particles from the solution as well as from other particulate systems. ${ }^{2-4}$ Moreover, clay particles are also used as carriers of pesticides, decolorizing agents, and environmental pollutants. ${ }^{5} \mathrm{We}$ demonstrated that the absolute number of MG molecules transferring from the PSS particles to the clay particles can be measured in real time. The effect of the clay particle density on the transfer kinetics was also observed. We also used the SHG method to study the molecular transfer of MG between PSS particles. Studying molecular transfer between the same kind of particles is not straightforward. Most of the present techniques rely on some careful calibration of signal changes upon molecular interaction with the microenvironment to distinguish the location of the molecules. ${ }^{6-8}$ When molecules transfer from particles to other particles of the same kind, the microenvironment remains the same. Therefore, the changes of signal are not easy to detect. The coherent nature of the SHG signal makes it a unique way to observe molecular transfer between like microparticles.

\footnotetext{
* To whom correspondence should be addressed.
}

\section{SCHEME 1}

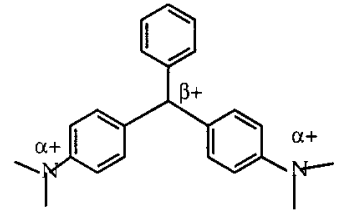

Second-harmonic generation (SHG) is a surface-specific second-order optical technique, ${ }^{6-9}$ which is described as electric dipole forbidden in centrosymmetric media. Because of the noncentrosymmetric nature of interfaces, SHG signal can be generated from the interfaces but not from the centrosymmetric bulk media. It has been proven that SHG is a powerful technique for probing kinetics and equilibrium at interfaces, including liquid/liquid, liquid/solid, liquid/vapor, solid/solid, and solid/ vapor interfaces. ${ }^{9-12}$

SHG has recently been shown to be a new method to probe microparticle surfaces. ${ }^{13}$ Though a colloidal microparticle such as a sphere or a disk is centrosymmetric, the local regions of the surfaces of microparticles are generally noncentrosymmetric. The second-harmonic field can be induced from the locally noncentrosymmetric surface upon irradiation of light. If the size of the microparticle is comparable to the wavelength of the light, the second-harmonic field from the different regions of the surface of the particle can add up coherently and give an SHG signal. Taking advantage of both the surface specificity and spectroscopic selectivity of SHG, it has been applied to study various microparticles suspended in liquid media including liposomes, ${ }^{15-17}$ emulsions, ${ }^{1,14}$ clay particles, ${ }^{18-19}$ polymer particles, $, 14,20$ semiconductor particles ${ }^{21}$, and metallic nanoparticles. ${ }^{22}$ A theory of SHG from the surface of small particles has been recently published. ${ }^{23}$

The polymer particles are polystyrene microspheres (PSS) of diameter $0.24 \pm 0.01 \mu \mathrm{m}$. They are negatively charged 
because of the sulfate groups on the surfaces. It has been demonstrated that when MG is adsorbed on PSS, a strong SHG signal is observed. ${ }^{1,13}$ It was shown in the earlier work ${ }^{13}$ that the adsorption free energy and number of adsorption sites of MG on PSS particles of diameter $1.0 \mu \mathrm{m}$ could be obtained from the adsorption isotherm measured by the SHG method.

The clay particles used are disk-shaped montmorillonite particles of diameter $0.5 \mu \mathrm{m}$ and thickness $0.01 \mu \mathrm{m} .{ }^{18-19}$ It was shown that the adsorptive and electrostatic properties ${ }^{18}$ as well as the rotational dynamics ${ }^{19}$ of the clay particles in aqueous solutions can be probed by the SHG method. Because montmorillonite has a structure that is centrosymmetric, ${ }^{5}$ there is no SHG generated from the bulk of the montmorillonite. A montmorillonite particle is composed of about 10 unit layers, and each layer consists of an octahedral sheet of alumina sandwiched between two tetrahedral sheets of silica. The basal surfaces of the particles are negatively charged because of isomorphous substitution of aluminum or silicon by other lower valence metal ions. The clay edge surfaces are positively charged in neutral or acidic environment because of the broken and hydrolyzed $\mathrm{Al}-\mathrm{O}$ and $\mathrm{Si}-\mathrm{O}$ bonds.

In situ studies of molecular transfer between microscopic particles can be studied by fluorescence methods, such as fatty acid transfer between liposomes, ${ }^{6}$ and bilirubin transfer between liposomes and proteins. ${ }^{7}$ In these studies, the molecules of interest are labeled by fluorescent probes, and the surfaces of either the donor or the acceptor particles are modified to interact with the transferring molecule to change the fluorescence quantum yield of the adsorbed fluorescent probe. ${ }^{6-8}$ These studies yield very useful information of transfer kinetics, but may suffer from the background of the fluorescence molecules present in the bulk solutions and the scattering of the excitation light. The systems may also be perturbed by the labeling of the acceptor microparticle surface.

SHG is a surface-specific technique, ${ }^{9-12}$ which responds to the molecules adsorbed on the surfaces of the colloidal particles. The unbound molecules in the bulk solution do not give an SHG signal. Therefore, no label molecule, quencher, shifting agent, nor modification of surface is needed. Because SHG is a coherent process, the SHG signal scales linearly with the microparticle density but quadratically with the population adsorbed on each of the microparticles. Thus, if the distribution of the same number of adsorbates among identical microparticles differs, the SHG signals will differ. Of course, the SHG method has its limitations. It is required that either the donor or the acceptor particles give a detectable SHG signal.

\section{Experimental Section}

The setup for the second-harmonic measurement is shown elsewhere. ${ }^{1,17,21}$ Briefly, it consists of a Ti:sapphire oscillator, which provides $100 \mathrm{fs}$ pulses at $846 \mathrm{~nm}$ at a repetition rate of $82 \mathrm{MHz}$. The fundamental light is focused into a rectangular 1 $\mathrm{cm}$ cell, and the second-harmonic photons at $423 \mathrm{~nm}$ are detected at $90^{\circ}$ or $0^{\circ}$ to the incident light by a single-photoncounting system. A monochromator was used to spectrally disperse the output signal.

Malachite green chloride purchased from Aldrich was used as received after checking its purity by HPLC. The polystyrene sulfate (PSS) particles used in the studies of transfer of MG from PSS to clay were of diameter $0.24 \pm 0.01 \mu \mathrm{m}$, and the PSS particles used in the studies of transfer of MG from PSS to PSS were of diameter $1.03 \pm 0.01 \mu \mathrm{m}$. They were purchased from Polysciences as an aqueous suspension, which was diluted in double-distilled water for the experiments. The clay sample of Na-montmorillonite was stored in $0.5 \mathrm{M} \mathrm{NaCl}$ solution until it was used. The suspension was centrifuged (15000 rpm for 5 $\mathrm{min}$ ), resuspended, and washed with double-distilled water until a negative chloride test was obtained with silver nitrate. The concentration of washed clay particles in aqueous solution was determined by a simple weighing method. All experiments were conducted at room temperature $22{ }^{\circ} \mathrm{C}$, and all the solutions were prepared in double-distilled water.

In the experiment of molecular transfer from the PSS particles to the clay particles, a $2.0 \mathrm{~cm}^{3}$ solution of the mixture of $\mathrm{MG}$ and PSS was prepared, and a $0.2 \mathrm{~cm}^{3}$ solution of the suspension of clay particles was rapidly injected into the $2.0 \mathrm{~cm}^{3}$ mixture. The SHG signal was monitored at a $0.1 \mathrm{~s}$ interval using the $90^{\circ}$ detection geometry. In the experiment of molecular transfer from PSS to PSS particles, a $1.5 \mathrm{~cm}^{3}$ of plain PSS was injected into an equal volume of an MG-PSS solution. The SHG signal was monitored at a $1.0 \mathrm{~s}$ interval using the forward-detection geometry. The injection and mixing were complete within a second. The time zero was set at the time of injection.

\section{Adsorption of MG on Polystyrene Particles and Clay Particles}

It was observed experimentally that the adsorption of MG on clay particles yields no SHG signal. It is known that the basal surfaces of the particles are negatively charged, but the edge surfaces are positively charged. ${ }^{5}$ The organic cations, MG, prefer to adsorb on the negatively charged basal plane. However, the top and bottom of the basal plane of a clay particle is separated by the thickness of the disk, $\sim 10 \mathrm{~nm}$, which is much smaller than the wavelength of the light. The second-harmonic field induced by the MG adsorbed on the opposite surfaces of the basal plane will be of opposite phase. Therefore, they cancel and give no SHG signal. It is also possible that MG molecules intercalate into the silica-alumina sheets of the particles. For the same reason, the second-harmonic fields induced from the intercalated MG will cancel, giving no overall SHG signal. ${ }^{19}$

An adsorption isotherm of MG on the PSS particles of diameter $0.24 \mu \mathrm{m}$ was obtained by measuring the secondharmonic field as a function of the bulk concentration of MG. The adsorption isotherm was fitted to the Langmuir model modified to include the depletion of MG in the bulk solution because of the adsorption which is described in details elsewhere. ${ }^{1,17}$ The adsorption equilibrium is treated as a reaction of the molecule (M) in the bulk solution with an empty site (ES) at the interface to give a filled site (FS):

$$
\mathrm{M}+\mathrm{ES} \underset{k_{-1}}{\stackrel{k_{1}}{\rightleftharpoons}} \mathrm{FS}
$$

At equilibrium, $\mathrm{d} N / \mathrm{d} t=0$, the following is obtained:

$$
K_{\mathrm{eq}}=\frac{k_{1}}{k_{-1}}=\frac{N}{(C-\rho N)\left(N_{\max }-N\right)}
$$

where $N$ is the number of molecules at the interface, that is, the number of filled sites; $N_{\max }$ is the total number of sites at the interface; $\rho$ is the density of the PSS microparticles; $C$ is the initially added concentration of $\mathrm{M} ; K_{\mathrm{eq}}$ is the equilibrium constant. For the PSS particles of diameter $0.24 \mu \mathrm{m}$, the adsorption equilibrium constant is found to be $4.2 \times 10^{6} \mathrm{M}^{-1}$, and the number of adsorption sites is found to be $1.6 \times 10^{5}$ per PSS particles. For the PSS particles of diameter $1.0 \mu \mathrm{m}$, the adsorption equilibrium constant reported previously was $3.0 \times$ 

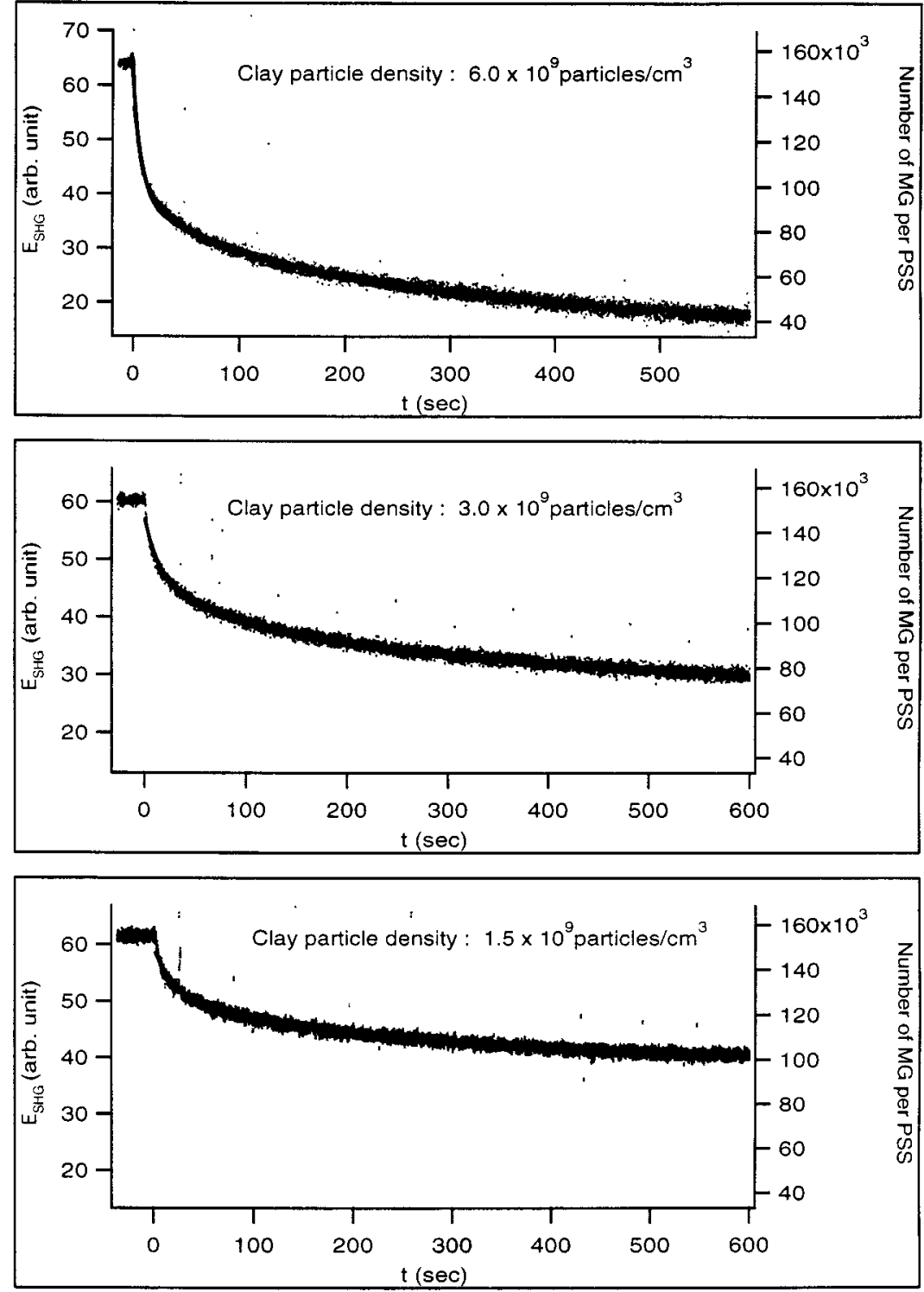

Figure 1. The decay of the second-harmonic field upon the addition of a $0.2 \mathrm{~cm}^{3}$ suspension of clay particles to the $2.0 \mathrm{~cm}^{3}$ mixture of PSS and MG to give the final density of clay particle (a) $6.0 \times 10^{9}$ particles $/ \mathrm{cm}^{3}$, (b) $3.0 \times 10^{9}$ particles $/ \mathrm{cm}^{3}$, and (c) $1.5 \times 10^{9} \mathrm{particles} / \mathrm{cm}^{3}$. The MG concentration is $9 \mu \mathrm{M}$, and the density of PSS particles is $3 \times 10^{10}$ particles $/ \mathrm{cm}^{3}$ after the addition of the clay particles. The solid lines are the double exponential fits. The fitting results are summarized in Table 1.

$10^{6} \mathrm{M}^{-1}$, and the number of adsorption sites was found to be $1.85 \times 10^{6}$ per PSS particles. ${ }^{1}$

\section{Transfer Kinetics of MG from PSS to Clay Particles}

The kinetics of MG transferring from PSS particles to clay particles can be obtained in real time. A suspension of clay particles was rapidly injected into a premixed PSS-MG solution (Scheme 2). The second-harmonic field is monitored as a function of time. The initial SHG signal before the mixing is due to the MG molecules adsorbed on the PSS particles. After the mixing, a decay of the second-harmonic field is observed, which is attributed to the adsorption of bulk MG onto clay followed by the desorption into the bulk solution of MG from the PSS particles to maintain equilibrium between MG on PSS with the bulk MG. The MG molecules remaining on the PSS particles give an SHG signal but those adsorbed on the clay particles do not. Figure 1 shows the kinetics data for different clay particle densities.

\section{SCHEME 2}

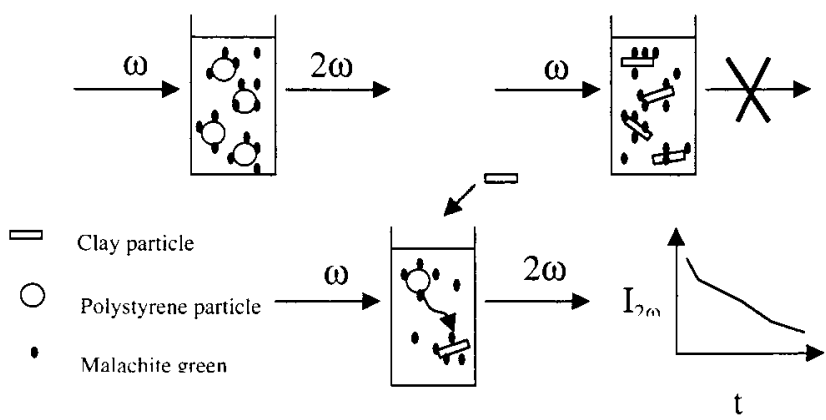

We found that the absolute number of adsorbates remaining on the PSS particles during the transfer processes can be measured in real time. Because of the coherent nature of the SHG signal, the second-harmonic fields induced from the molecules adsorbed on the same particle add up coherently, whereas the second-harmonic fields generated from different 


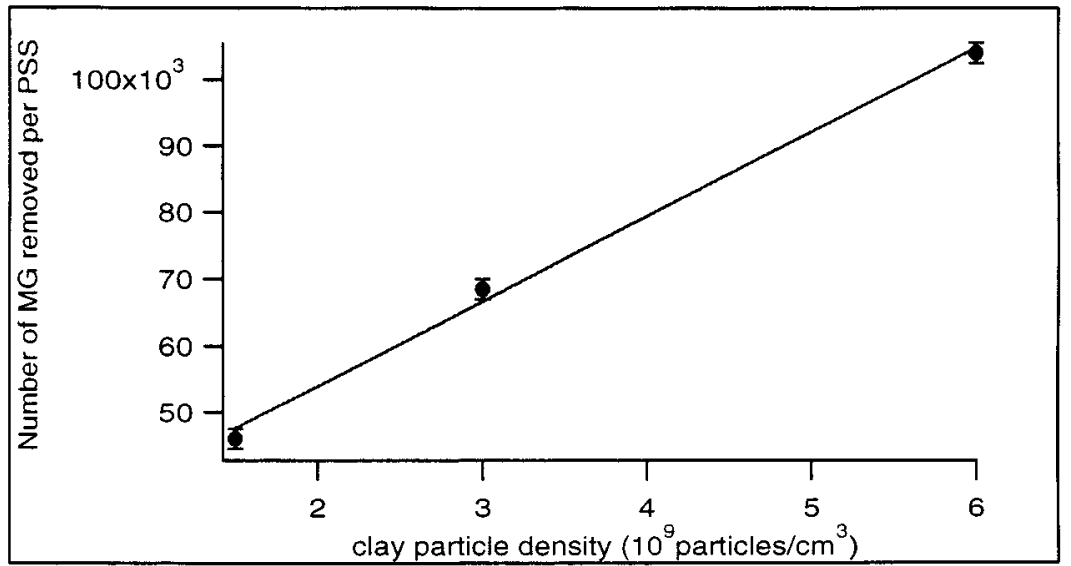

Figure 2. The number of MG removed per PSS particle by the clay particles is plotted as a function of the particle density of clay, yielding a linear dependence. The final MG concentration is $9 \mu \mathrm{M}$, and the particle density of PSS is $3 \times 10^{10}$ particles $/ \mathrm{cm}^{3}$.

TABLE 1: Effect of Particle Density on the Transfer Kinetics of MG from the PSS Particles to the Clay Particles ${ }^{a}$

\begin{tabular}{ccrrrr}
\hline particle density $\left(10^{9}\right.$ particles $\left./ \mathrm{cm}^{3}\right)$ & \multicolumn{1}{c}{$a_{0}$} & \multicolumn{1}{c}{$a_{1}$} & \multicolumn{1}{c}{$a_{2}$} & $\tau_{2}(\mathrm{~s})$ \\
\hline 1.5 & $47.1 \pm 0.8$ & $9.7 \pm 0.1$ & $8.7 \pm 0.2$ & $281 \pm 19$ & $25.3 \pm 1.6$ \\
3.0 & $37.3 \pm 0.1$ & $13.2 \pm 0.1$ & $11.7 \pm 2.3$ & $217 \pm 4$ & $16.9 \pm 0.4$ \\
6.0 & $28.9 \pm 0.1$ & $15.8 \pm 0.1$ & $20.3 \pm 2.2$ & $182 \pm 2$ & $10.0 \pm 0.2$
\end{tabular}

${ }^{a}$ The decays of SH field are fitted to double exponential function, $a_{0}+a_{1} \exp \left(-\mathrm{t} / \tau_{1}\right)+a_{2} \exp \left(-\mathrm{t} / \tau_{2}\right)$. The particle density of PSS particles after the addition of the clay particles was $3 \times 10^{10}$ particles $/ \mathrm{cm}^{3}$.

particles add up incoherently when the particles are separated by a distance longer than the coherence length of the process. ${ }^{12}$ Therefore, the second-harmonic intensity scales linearly with the PSS particle density, but quadratically with the surface adsorbates population.

$$
I_{\mathrm{SHG}} \propto \rho N^{2}
$$

where $\rho$ is the PSS particle density, and $N$ is the number of adsorbed molecules on an individual PSS particle. In the experiments, the particle density of the $0.2-\mu$-sized PSS is $3 \times$ $10^{10}$ per $\mathrm{cm}^{3}$ giving an average separation of $3 \mu$. It is calculated that the coherence length of the SHG process with detection at $90^{\circ}$ to the incident beam is in the submicron range. ${ }^{25}$ An equation relating the surface population, particle density, and the measured second-harmonic field before and after the addition of clay suspension can be written as

$$
\frac{E_{\mathrm{SHG}}(t)}{E_{\mathrm{SHG}}(t<0)}=\sqrt{\frac{I_{\mathrm{SHG}}(t)}{I_{\mathrm{SHG}}(t<0)}}=\left(\frac{\rho(t>0)}{\rho(t<0)}\right)^{1 / 2}\left(\frac{N(t)}{N(t<0)}\right)
$$

Therefore,

$$
N(t)=N(t<0) \frac{E_{\mathrm{SHG}}(t)}{E_{\mathrm{SHG}}(t<0)}\left(\frac{\rho(t<0)}{\rho(t>0)}\right)^{1 / 2}
$$

where $E_{\mathrm{SHG}}$ is the second-harmonic field, $I_{\mathrm{SHG}}$ is the measured SHG intensity, $t=0$ refers to the time at which the clay particles are added to the PSS-MG mixtures, and $\rho(t>0)$ is the PSS particle density after addition of a small volume of clay particles.

The surface population of MG on the PSS during the transfer process, $N(t)$, can be obtained by eq 4 . First, we need to obtain the surface population of MG on the PSS before the addition of clay, $N(t<0)$. The MG concentration before the addition of the clay particles is $10 \mu \mathrm{M}$, and the PSS particle density is 3.3 $\times 10^{10}$ particles $/ \mathrm{cm}^{3}$. Using the adsorption equilibrium constant, $4.23 \times 10^{6} \mathrm{M}^{-1}$, obtained from the adsorption isotherm of $\mathrm{MG}$ on PSS particles, we calculated that $N(t<0)$ is $1.47 \times 10^{5}$ per
PSS. Second, we need to consider the changes of the PSS particle density because of the addition of the clay suspension. The ratio of $\rho(t<0)$ to $\rho(t>0)$ is $1.1: 1$ because $0.2 \mathrm{~cm}^{3}$ clay particle suspension was added to $2.0 \mathrm{~cm}^{3} \mathrm{MG}-\mathrm{PSS}$ solution in the experiments. The number of MG molecules per PSS particle during the transfer process can be found by measuring the second-harmonic field, $E_{\mathrm{SHG}}(t)$ and $E_{\mathrm{SHG}}(t<0)$, as shown in Figure 1. The surface populations of MG on the PSS particles are plotted on the right axes in Figure 1. The number of MG removed per PSS particle, $N(0)-N(\infty)$, depends linearly on the particle density of clay as shown in Figure 2.

The effects of the clay particle density on the transfer kinetics were examined. The density of the clay particles was varied from 6.0 to $1.5 \times 10^{9}$ particles $/ \mathrm{cm}^{3}$. The decay of the secondharmonic field is fitted empirically to double exponential functions shown as a solid line in Figure 1. The fitting results are summarized in Table 1. Both the slow and the fast decay constants, $1 / \tau_{1}$ and $1 / \tau_{2}$, scale linearly with the particle density as shown in Figure 3.

Before the addition of the clay particles, an adsorption equilibrium of MG on the PSS surfaces is established. At 10 $\mu \mathrm{M}$ of $\mathrm{MG}$ concentration and particle density of $3.3 \times 10^{10}$ particle $/ \mathrm{cm}^{3}$, the adsorption sites on the PSS particles are saturated. It can be calculated from the equilibrium equation that the concentration of free MG in the solution is $2.1 \mu \mathrm{M}$. When the clay particles are added to the PSS-MG solution, the free MG molecules diffuse and adsorb onto the surface of clay particles leading to a decrease of the bulk MG concentration. Immediately, the bulk MG concentration decreases, which shifts the adsorption equilibrium of MG on PSS, leading MG molecules to desorb from the PSS particles, and consequently to adsorb onto the clay particles until equilibrium is reached. It is also possible that the transfer of MG molecules can happen during the collision of the PSS particles.

Among the possible mechanisms are (1) the collision mechanism by which the transfer kinetics of MG is determined in part by the collision between clay and PSS particles and (2) desorption-adsorption mechanism for which the transfer kinet- 

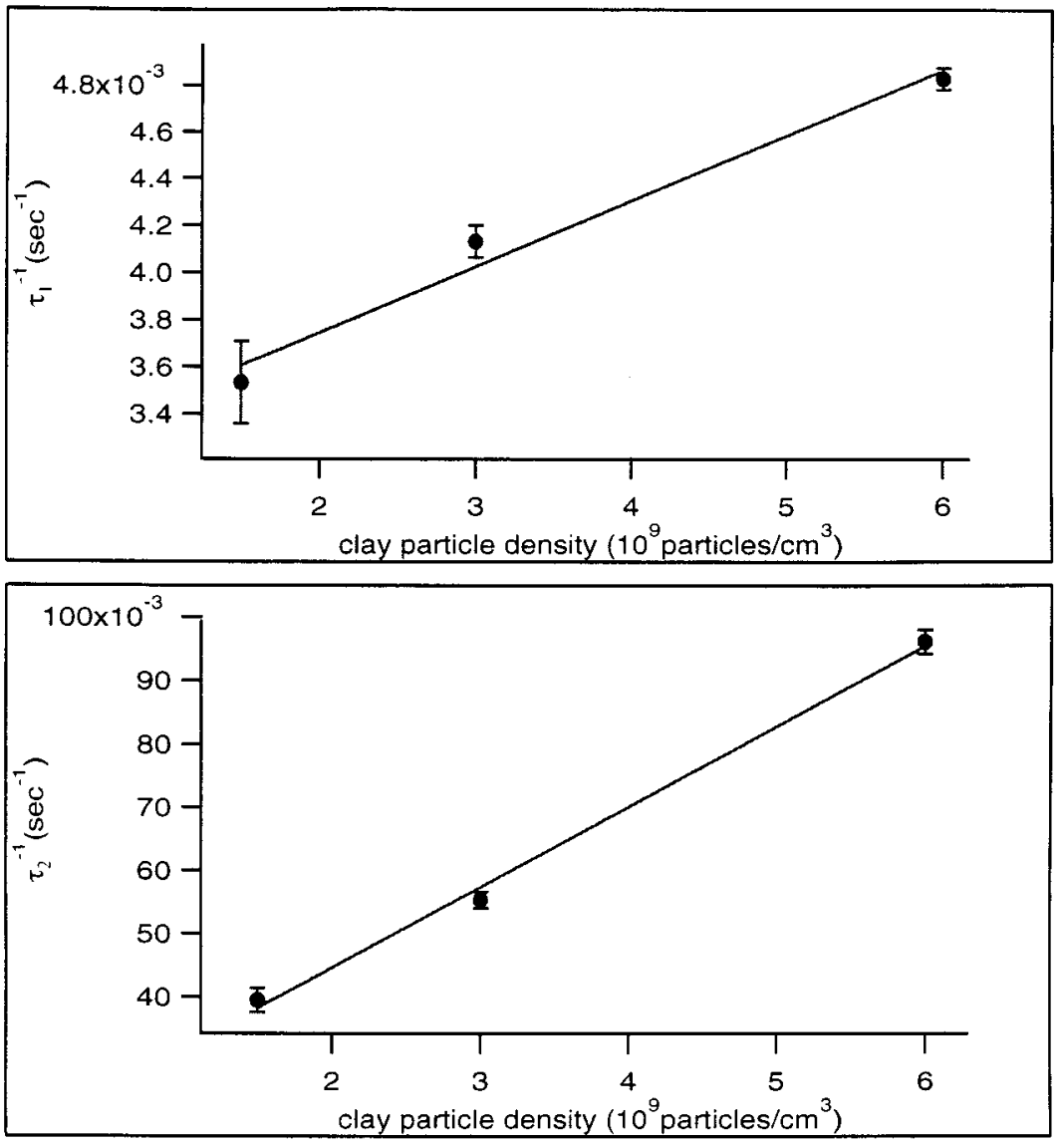

Figure 3. The rate constants of the slow and fast decay, $\tau_{1}{ }^{-1}$ and $\tau_{2}^{-1}$ respectively, are plotted as a function of the particle density of clay, both yielding a linear dependence.

ics is determined by the desorption of MG from the PSS particles followed by an adsorption onto the clay surfaces.

\section{Transfer Kinetics of MG between PSS Particles}

Because of the coherent nature of the SHG signal, ${ }^{9-13}$ the SHG method can be used to probe the molecular transfer between colloidal particles of the same kind. Second-harmonic intensity scales linearly with particle density, but quadratically with surface population as described in eq 2. After the plain acceptor PSS particles are added to the premixed MG-PSS solution, the redistribution of MG among the donor and acceptor PSS will lead to a reduction in the SHG signal. This is because the signal depends quadratically on the MG population on the PSS particles, and the MG population per PSS decreases with interparticle transfer. The transfer of molecules adsorbed on colloids of the same kind is difficult to study by conventional methods because there must be a change in the microenvironment to distinguish the location of the adsorbed molecules.

Figure 4 shows the decay of the SHG intensity upon addition of plain PSS particles to the mixture of PSS and MG solution. A $1.5 \mathrm{~mL}$ PSS-MG solution was first prepared at particle density of $4 \times 10^{8}$ particles $/ \mathrm{cm}^{3}$ and MG concentration of 0.12 $\mu \mathrm{M}$. An equal volume of plain PSS at the same particle density was injected into the PSS-MG solution. The intensity immediately dropped to one-half of the initial signal and then exponentially decayed to an equilibrium value. The final MG per PSS is one-half of its initial value at $t=0$, and the SHG intensity is one-fourth of the signal at $t<0$.
TABLE 2: Rate Constants (Adsorption, $k_{1}$, and Desorption, $\boldsymbol{k}_{-1}$ ) for MG Transfer between PSS Particles Are Obtained from SHG Kinetics Measurements ${ }^{a}$

\begin{tabular}{lcclccc}
\hline$C(\mu \mathrm{M})$ & $\mathrm{C}_{\mathrm{b}}^{\mathrm{i}}(\mu \mathrm{M})$ & $C_{\mathrm{b}}^{\mathrm{f}}(\mu \mathrm{M})$ & $C_{\mathrm{b}}(\mu \mathrm{M})$ & $\tau_{1}(\mathrm{~s})$ & $k-1\left(\mathrm{~s}^{-1}\right)$ & $k_{1}\left(\mathrm{~s}^{-1} \mathrm{M}^{-1}\right)$ \\
\hline 0.045 & 0.010 & 0.010 & 0.010 & 36 & 0.014 & $4.1 \times 10^{4}$ \\
0.060 & 0.014 & 0.013 & 0.0134 & 41 & 0.012 & $3.5 \times 10^{4}$ \\
0.080 & 0.018 & 0.018 & 0.018 & 37 & 0.013 & $4.0 \times 10^{4}$ \\
0.12 & 0.029 & 0.027 & 0.028 & 31 & 0.015 & $4.5 \times 10^{\mathrm{Y}}$
\end{tabular}

${ }^{a} C$ is the total MG concentration at $t \geq 0 ; C_{b}{ }^{i}$ is the initial bulk MG concentration at $t=0, C_{b}{ }^{i}$ is the final bulk MG concentration at $t \rightarrow$ $\infty ; \mathrm{C}_{\mathrm{b}}$ is the average of initial and final MG concentration; $\tau$ is the decay time obtained from fitting the kinetic data. Rate constants $k_{1}$ and $k_{-1}$ are obtained by equation (13) and $\mathrm{K}_{\mathrm{eq}}=k_{1} / k_{-1}$.

When an equal volume of plain PSS is added to the PSSMG mixture, the particle density of PSS-MG, $\rho$, is halved before any transfer of MG. Therefore, the observed SHG intensity is half of the original, $t<0$, value.

$$
I_{\mathrm{SHG}}(t=0) \propto \frac{\rho}{2} N^{2}
$$

Then, the SHG intensity decreases until a new equilibrium is established in which the MG molecules are equally distributed on the surfaces of the PSS particles. It is calculated from the adsorption equilibrium equation and illustrated in Table 2 that after the instant halving at $t=0$, the change of the number of MG molecules in the bulk solution is negligible, that is, $<6 \%$ in the maximum case. Because the number of MG per PSS 


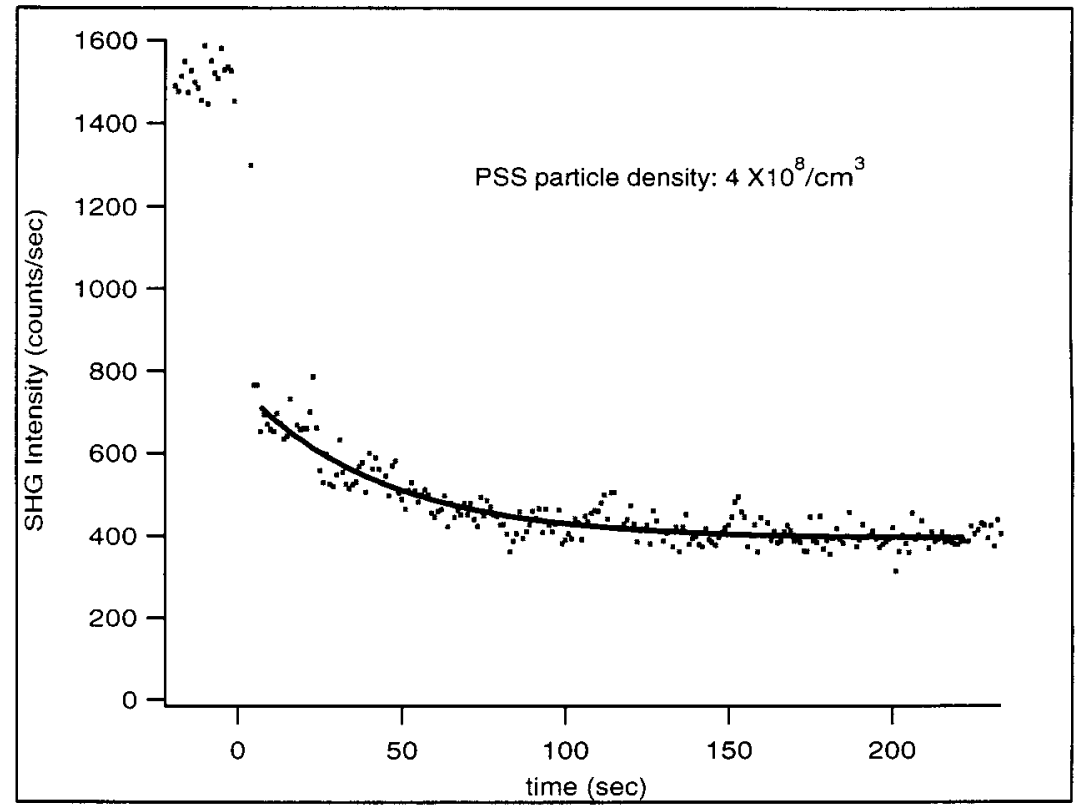

Figure 4. A $1.5 \mathrm{~mL}$ of plain PSS particles is injected to a $1.5 \mathrm{~mL} \mathrm{MG-PSS} \mathrm{solution.} \mathrm{The} \mathrm{diameter} \mathrm{of} \mathrm{the} \mathrm{PSS} \mathrm{particles} \mathrm{is} 1.0 \mu$, and the particle density is $4 \times 10^{8} / \mathrm{cm}^{3}$. The MG total concentration is $0.06 \mu \mathrm{M}$ at $t \geq 0$. The SHG intensity immediately drops to a half of the original signal, followed by a single-exponential decay. The final intensity is one-fourth of the initial intensity. The decay time is found to be $40 \mathrm{~s}$.

particle is reduced from $N$ at $t<0$ to $N / 2$ at the new equilibrium at $t \rightarrow \infty$, the final SHG signal is

$$
I_{\mathrm{SHG}}(t \rightarrow 0) \propto \rho\left(\frac{N}{2}\right)^{2}
$$

Consequently, the ratio of the second-harmonic intensities at $t$ $<0, t=0$, and $t \rightarrow \infty$ is $4: 2: 1$. This is observed experimentally as shown in Figure 4, where the second-harmonic intensities are $1532 \pm 40$ counts per sec at $t<0,766$ counts per sec at $t$ $=0$, and $391 \pm 27$ counts per second at $t \rightarrow \infty$.

A model is used to describe the transfer of MG between the PSS particles by which the desorption rate constant $k_{-1}$ of MG from PSS can be extracted from the kinetic data. With the adsorption equilibrium constant known from the adsorption isotherm, the adsorption rate constant $k_{1}$ can also be found.

In the model, we consider two kinds of particles: P1 with MG initially adsorbed on the surface and P2 initially without MG adsorbed on the surface. Both particle densities are halved to $\rho / 2$ after the mixing of P2 with P1. The adsorption equilibrium initially established on particle P1 is disturbed by the introduction of new empty sites on P2. The bulk MG molecules move to the new sites on $\mathrm{P} 2$, which causes a desorption of MG from P1 to the bulk solution. This process continues until the new equilibrium is established.

At time $t$ after the mixing, the intensity of the SHG signal is

$$
I_{\mathrm{SHG}}(t) \propto \frac{\rho}{2}\left[N_{1}(t)\right]^{2}+\frac{\rho}{2}\left[N_{2}(t)\right]^{2}
$$

where $N_{1}(t)$ is the number of $\mathrm{MG}$ on $\mathrm{P} 1$ and $N_{2}(t)$ is the number of MG on $\mathrm{P} 2$ at time $t$.

By mass conservation, we have

$$
C=\frac{\rho}{2} N_{1}(t)+\frac{\rho}{2} N_{2}(t)+C_{\mathrm{b}}(t)
$$

where $C$ is the total $\mathrm{MG}$ concentration in the system, and $C_{\mathrm{b}}$ is the concentration of MG molecules free in the bulk solution. The kinetic equations are

$$
\begin{gathered}
\frac{\mathrm{d} N_{1}}{k_{-1} \mathrm{~d} t}=-N_{1}+K_{\mathrm{eq}} C_{\mathrm{b}}\left(N_{\mathrm{max}}-N_{1}\right)=K_{\mathrm{eq}} C_{\mathrm{b}} N_{\mathrm{max}}- \\
\left(1+K_{\mathrm{eq}} C_{\mathrm{b}}\right) N_{1}=A-B N_{1} \\
\frac{\mathrm{d} N_{2}}{k_{-1} \mathrm{~d} t}=-N_{2}+K_{\mathrm{eq}} C_{\mathrm{b}}\left(N_{\max }-N_{2}\right)=K_{\mathrm{eq}} C_{\mathrm{b}} N_{\max }- \\
\left(1+K_{\mathrm{eq}} C_{\mathrm{b}}\right) N_{2}=A-B N_{2} \\
\frac{\mathrm{d} C_{\mathrm{b}}}{\mathrm{d} t}=-\left(\frac{\mathrm{d} N_{1}}{\mathrm{~d} t}+\frac{\mathrm{d} N_{2}}{\mathrm{~d} t}\right) \approx 0
\end{gathered}
$$

where $A=K_{\text {eq }} C_{\mathrm{b}} N_{\text {max }}, B=1+K_{\text {eq }} C_{\mathrm{b}}$. The bulk MG concentration is approximately constant as discussed earlier.

At time $t=0$, that is, immediately after the dilution,

$$
\begin{array}{r}
C(0)=\frac{1}{2} C(t<0), C_{\mathrm{b}}(0)=\frac{1}{2} C_{\mathrm{b}}(t<0), N_{2}(0)=0, N_{1}(0)= \\
N_{1}(t<0)
\end{array}
$$

At $t \rightarrow \infty$, that is, at the newly established equilibrium,

$$
N_{1}(\infty)=N_{2}(\infty)=\frac{K_{\mathrm{eq}} C_{\mathrm{b}}(\infty) N_{\mathrm{max}}}{\left[1+K_{\mathrm{eq}} C_{\mathrm{b}}(\infty)\right]}=\frac{A}{B}
$$

With the steady-state approximation, $\mathrm{d} C_{\mathrm{b}} / \mathrm{d} t \approx 0$, both $A$ and $B$ can be treated as constants. Therefore, the differential kinetic equations can be integrated and solved analytically.

$$
\int_{N_{1}(0)}^{N_{1}(t)} \frac{\mathrm{d} N_{1}}{A-B N_{1}}=\int_{0}^{t} \mathrm{~d}\left(k_{-1} t\right)
$$

The results are 


$$
\begin{gathered}
N_{1}(t)=\left[-\frac{A}{B}+N_{1}(0)\right] e^{-\mathrm{k}_{-1} B t}+\frac{A}{B} \\
N_{2}(t)=-\frac{A}{B} e^{-\mathrm{k}_{-1} B t}+\frac{A}{B}
\end{gathered}
$$

Therefore,

$$
\frac{I_{\mathrm{SHG}}(t)}{I_{\mathrm{SHG}}(0)}=\frac{N_{1}(t)^{2}+N_{2}(t)^{2}}{N_{1}(0)^{2}}=\frac{1}{2} e^{-2 \mathrm{k}_{-1} B t}+\frac{1}{2}
$$

The apparent decay time constant $\tau$ for $I_{\text {SHG }}$ will be $\left[-2 k_{-1}(1\right.$ $\left.\left.+K_{\mathrm{eq}} C_{\mathrm{b}}\right)\right]^{-1}$.

The above model holds only when (1) the steady-state approximation is valid and (2) the desorption-adsorption is the dominant pathway for the molecular transfer. To satisfy the first condition, the total MG concentration has to be low so that the change of $C_{\mathrm{b}}$ becomes negligible. To satisfy the second condition, the particle density has to be low so that molecular transfer via "collision" of the microparticles is not ratedetermining. The average separation between PSS particles is $13 \mu \mathrm{m}$. The collision time scale of $1.0 \mu \mathrm{m}$ PSS microparticles is calculated to be $1400 \mathrm{~s}$, which is orders of magnitude longer than the measured decay time.

The transfer kinetics was studied at different final $\mathrm{MG}$ concentrations of $0.045,0.06,0.08$, and $0.12 \mu \mathrm{M}$. The desorption constant, $k_{-1}$, can be extracted from the kinetic data using eq 13. With the adsorption equilibrium constant known from the adsorption isotherm, one can calculate the adsorption rate constant, $k_{1}$. The values of the calculated $k_{-1}$ and $k_{1}$ are listed in Table 2. From the table, we can also see that the change of bulk MG concentration during the transfer process is $6 \%$ at most for the case of $0.12 \mu \mathrm{M}$. The steady-state approximation is valid.

\section{Conclusion}

These studies demonstrate the applicability of the SHG method in studying the kinetics of molecular transfer between microparticles suspended in aqueous solution. It was found that the transfer of MG from the PSS particles to clay particles can be monitored in real time by measuring the surface population of MG remaining on the PSS particles. We observed that the kinetics depends on the particle density of the clay. A biexponential decay was observed with decay times of $17 \mathrm{~s}$ and 217 $\mathrm{s}$ for a clay particle density of $3 \times 10^{9} / \mathrm{cm}^{3}$. This method was also applied to study the molecular transfer among PSS particles. A model was used to describe the transfer process, from which the desorption and adsorption rate constants were found to be $0.013 \mathrm{~s}^{-1}$ and $4 \times 10^{4} \mathrm{~s}^{-1} \mathrm{M}^{-1}$ respectively.

Acknowledgment. We would like to thank Prof. C. Johnston at Purdue University for providing us the clay sample of $\mathrm{Na}$ montmorillonite. We thank the National Science Foundation and the Division of Chemical Science, Office of Basic Sciences of the Department of Energy for their generous support.

\section{References and Notes}

(1) Wang, H.; Yan, E. C. Y.; Liu, Y.; Eisenthal, K. B. J. Phys. Chem $B$ 1998, 102, 4446.

(2) Jenne, E. A. Adsorption of metals by Geomedia; Academic Press: New York, 1998. 153.

(3) Bargier, F.; Duc. G.; Petit-Ramel, M. Colloids Surf., A 2000, 166,

(4) Comstock, M. J. Sources and Fates of Aquatic Poluutants; American Chemical Society: Washington, D. C., 1987.

(5) VanOlphen, H. An Introduction to Clay Colloid Chemistry; Wiley: New York, 1963.

(6) Kleinfeld, A. M.; Storch, J. Biochemistry 1992, 32, 2053.

(7) Noy, N.; Leonard, L.; Azkim, D. Biophys. Chem. 1992, 42, 177

(8) Nichols, J. W.; Pagano, R. E. Biochemistry 1982, 21, 1720. 1984.

(9) Shen, Y. R. The Principles of Nonlinear Optics; Wiley: New York,

(10) Eisenthal, K. B. Chem. Rev. 1996, 96, 1343.

(11) Corn, R. M.; Higgins, D. A. Chem. Rev. 1994, 94, 107.

(12) Richmond, G.; Robinson, J. M.; Shannon, V. L. Prog. Surf. Sci. 1988, 28,1 .

(13) Wang, H.; Yan, E. C. Y.; Borguet, E.; Eisenthal, K. B. Chem. Phys. Lett. 1996, 259, 15.

(14) Yan, E. C. Y, Liu, Y.; Eisenthal, K. B. J. Phys. Chem. B 1998, 102,6331 .

(15) Srivastava, A.; Eisenthal, K. B. Chem. Phys. Lett. 1998, 292, 345.

(16) Yan, E. C. Y.; Eisenthal, K. B. Biophys. J. 2000, 79, 898.

(17) Liu, Y.; Yan, E. C. Y.; Eisenthal, K. B. Biophys. J. 2001, 80, 1004

(18) Yan, E. C. Y.; Eisenthal, K. B. J. Phys. Chem. B 1999, 103, 6056.

(19) Yan, E. C. Y.; Eisenthal, K. B. J. Phys. Chem. B 2000, 104, 6686.

(20) Wang, H. F.; Troxler, T.; Yeh, A. G.; Dai, H. L. Langmuir 2000 , 16,2475

(21) Liu, Y.; Dadap, J. I.; Zimdars, D.; Eisenthal, K. B. J. Phys. Chem. $B$ 1999, 103, 2480 .

(22) Yan, E. C. Y.; Eisenthal, K. B. Unpublished results 2000.

(23) Dadap, J. I.; Shan, J.; Eisenthal, K. B.; Heinz, T. F. Phys. Rev. Lett. 1999, 83, 4045 . 